\title{
Miranda
}

Revue pluridisciplinaire du monde anglophone /

Multidisciplinary peer-reviewed journal on the English-

speaking world

$18 \mid 2019$

Guerre en poésie, poésie en guerre

\section{Reparadise de Gwenaël Morin ou la dépolitisation d'un spectacle historiquement subversif.}

Critique de Reparadise de Gwenaël Morin

\section{Camille Mayer}

\section{(2) OpenEdition}

\section{Journals}

Édition électronique

URL : http://journals.openedition.org/miranda/18253

DOI : 10.4000/miranda.18253

ISSN : 2108-6559

Éditeur

Université Toulouse - Jean Jaurès

Référence électronique

Camille Mayer, «Reparadise de Gwenaël Morin ou la dépolitisation d'un spectacle historiquement subversif. », Miranda [En ligne], 18 | 2019, mis en ligne le 17 avril 2019, consulté le 16 février 2021. URL : http://journals.openedition.org/miranda/18253; DOI : https://doi.org/10.4000/miranda.18253

Ce document a été généré automatiquement le 16 février 2021.

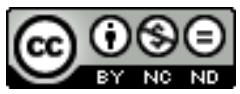

Miranda is licensed under a Creative Commons Attribution-NonCommercial-NoDerivatives 4.0 International License. 


\section{Reparadise de Gwenaël Morin ou la dépolitisation d'un spectacle historiquement subversif.}

Critique de Reparadise de Gwenaël Morin

\section{Camille Mayer}

\section{Re-Paradise: lien internet}

1 http://www.nanterre-amandiers.com/2017-2018/re-paradise/

\section{Re-Paradise: distribution}

Dates : 4, 5, 11, 12, 18, 19, 25, 26 mai 2018

Lieu : Théâtre Amandiers-Nanterre, Atelier

Durée : $1 \mathrm{~h} 50$

Spectacle de Gwénaël Morin à partir de Paradise Now, création collective du Living Theatre (retranscription originale de la pièce : Judith Malina, Julian Beck; traduction en français : Elsa Rooke)

Avec : Isabelle Angotti, Lluis Ayet Puigarnau, Gaël Baron, Elsa Bouchain, Michael Comte, Anne de Queiroz, Giulia Deline, Vincent Deslandres, Jean-Charles Dumay, Julian Eggerickx, Jonathan Foussadier, Cecilia Gallea, Alyse Gaultier, Gabrier, Gauthier, Léo Gobin, Jules Guittier, Barbara Jung, Manu Laskar, Victor Lenoble, Natacha Mendès, Nicole Mersy Ortega, Elsa Michaud, Julien Michel, Viviana Moin, Olga Mouak, Perle Palombe, Gianfranco Poddighe, Ulysse Pujo, Lison Rault, Thierry Raynaud, Richard Sammut, Mayya Sanbar, Brahim Tekfa, Thomas Tressy, Gaetan Vourc'h, Marc Zammit. 


\section{Re-Paradise de Gwenaël Morin ou la dépolitisation d'un spectacle historiquement subversif.}

7 En mai 2018, Gwenaël Morin, metteur en scène et alors directeur du théâtre du Point du Jour de Lyon, décide de mettre en scène Re-Paradise à partir de l'emblématique Paradise Now du Living Theater. Paradise Now avait été créé en 1968 par la troupe anarchiste américaine lors de sa tournée en Europe, plus spécifiquement pour sa venue lors de la $22^{e}$ édition du festival d'Avignon. Chaque troupe invitée devait présenter deux spectacles ayant déjà tourné et une création. Le Living fit le choix de jouer Mysteries and smaller pieces, Antigone et créa pour l'occasion Paradise Now.

Ce spectacle, éminemment politique et subversif, fit scandale et cristallisa notamment les revendications de la révolution de juillet $1968^{1}$. Cinquante ans plus tard, Gwenaël Morin, sur l'invitation de Philippe Quesne ${ }^{2}$ à participer au festival « Mondes possibles » du Théâtre Nanterre-Amandiers, propose de jouer à nouveau ce spectacle qu'il nomme Re-Paradise. Huit représentations auront lieu au théâtre Nanterre-Amandiers du 4 au 26 mai 2018, et une au Théâtre du Point du Jour le 21 juillet 2018 pour la clôture du théâtre - Gwenaël Morin quittant ses fonctions de directeur de structure. Si le metteur en scène a l'habitude de jouer des classiques de l'histoire du théâtre, il se confronte avec Re-Paradise à des problématiques plus importantes que pour des tragédies grecques ou des pièces de théâtre classiques ${ }^{3}$.

La première difficulté semble être celle de l'écriture dramaturgique. Les écrits ou propos du Living Theatre sur le spectacle sont relativement précis et accessibles : La vie du théâtre sont des mémoires de Julian Beck ${ }^{4}$, l'ouvrage de Jean-Jacques Lebel, Entretiens avec le Living Theater ${ }^{5}$ présente notamment les difficultés de la création à Cefalu, et Stéphanette Vendeville publie en 2007 Le Living Theatre, de la toile à la scène ${ }^{6}$ qui explique en détail la construction de Paradise Now. Gwenaël Morin s'empare de la version anglaise du spectacle, retranscrite par le Living Theater lui-même sous un titre éponyme ${ }^{7}$ et en commande une traduction en français, qu'il distribuera aux spectateur.ice.s au début de la représentation.

10 Le spectacle de 2018 est monté de façon similaire à la création originale. Paradise Now est pensé comme un voyage, pour les comédien.ne.s comme pour les spectateur.ice.s, vers la Révolution permanente, la Belle Révolution Anarchiste Non-Violente ${ }^{8}$. Cette pièce est construite en huit échelons : l'échelon du Bien et du Mal, l'échelon de la Prière, l'échelon de l'Enseignement, l'échelon de la Voie, l'échelon de la Rédemption, l'échelon de l'Amour, l'échelon du Ciel et de la Terre et l'échelon de Dieu et de l'Homme. Chaque échelon est lui-même constitué d'un Rite, d'une Vision et d'une Action, qui mènent à la réussite d'un aspect de la Révolution. Les rites sont des « cérémonies/rituels physiques/spirituels qui culminent dans un Flash. Les visions sont cérébrales [...] images, symboles, rêves ». Les rites et les visions, joués par les comédien.ne.s, provoquent une conscience qui mène à l'action de la part des spectateur.ice.s, aidés par les artistes. Bien que des lieux soient spécifiés pour les actions, celles-ci mènent à des révolutions dans l'ici et le maintenant. Gwenaël Morin a respecté la partition retranscrite par Julian Beck et Judith Malina. Il a même utilisé des lumières, contrairement à son habitude, afin de conserver les ambitions de la pièce. Les couleurs vont de l'obscurité à la clarté. Elles permettent l'unification des consciences réunies lors de la représentation. À chaque échelon est attribuée une couleur à laquelle sera ajoutée de la lumière blanche lors de l'action, pour augmenter l'effet de l'« ici et 
maintenant ». La lumière blanche est toujours tournée vers le public. Pour Gwenaël Morin comme pour le Living Theatre, le spectacle se termine dans la rue, « le théâtre est dans la rue. La rue appartient aux gens. Libérez le théâtre. Libérez la rue. Commencez » la Révolution.

Fig. 1

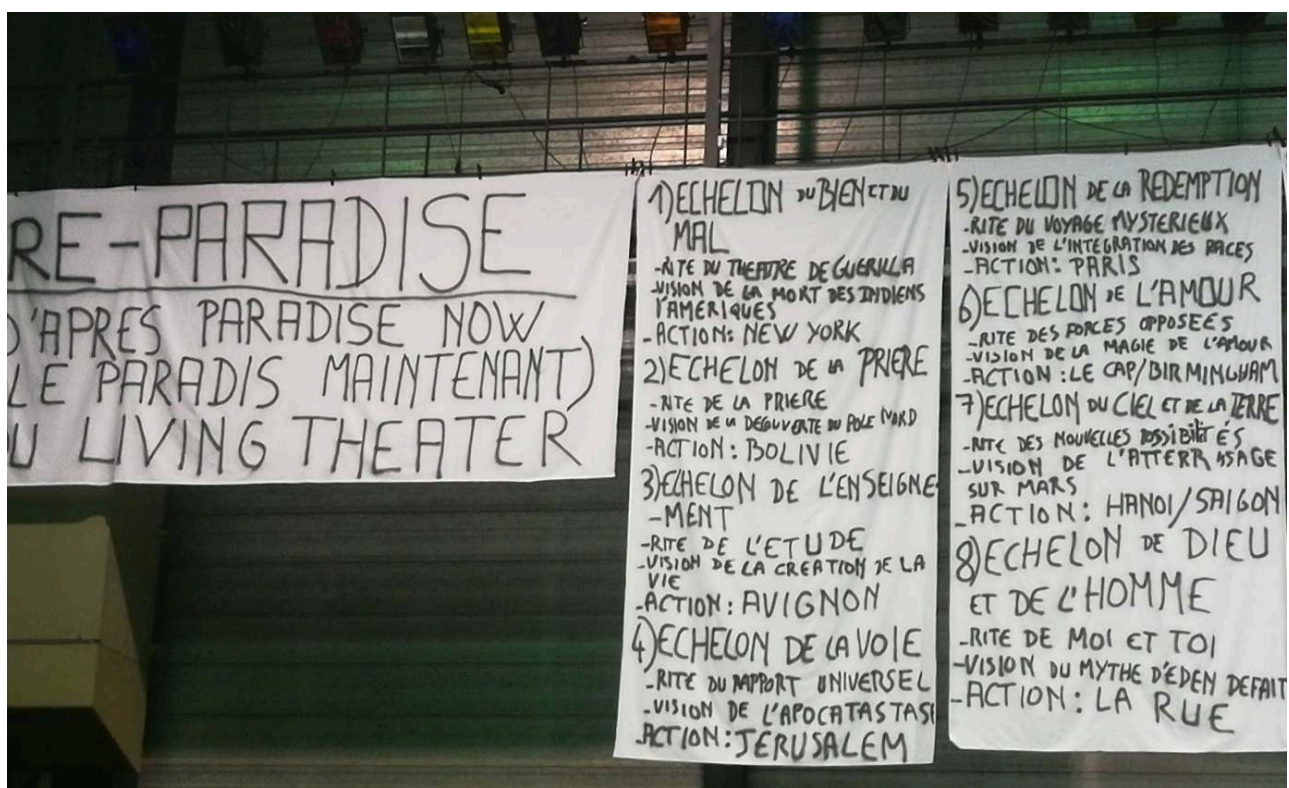

TENTURES ACCROCHÉES SUR UN PAN DU MUR DU THÉÂTRE NANTERRE-AMANDIERS, REPRENANT LE DÉROULÉ dU SPECTACLE AVEC SES HUIT ÉCHELONS ET, POUR CHACUN D'ENTRE EUX, LE RITE, LA VISION ET L'ACTION.

Crédit photo : Camille Mayer.

11 Si la version de Gwenaël Morin est fidèle à l'originale, c'est en réalité au niveau du processus de création que des modifications ont été faites, et que tout semble se jouer. Sa troupe n'est composée que de douze comédien.ne.s lorsqu'elle se lance dans ce projet. Le spectacle du Living Theatre en compte trente-six, qui vivent en communauté anarchiste depuis des années et ont effectué ensemble de multiples créations. Au Living Theatre, les répétitions sont journalières, les expérimentations théâtrales : « le Living n'est rien d'autre qu'un de ces groupes, une de ces communautés, vouées à l'étude des problèmes posés par l'expression théâtrale. [...] C'est comme cela que nous apprenons vraiment, car il y a initiation et transmission de la connaissance en même temps qu'expérimentation pratique de celle-ci ${ }^{9}$ ». La création de Paradise Now est collective, libre de droits pour n'importe quelle communauté qui souhaiterait la jouer ${ }^{10}$. C'est un aboutissement du travail communautaire et de la mise en place des principes anarchistes de non-domination et de déhiérarchisation. Dans un entretien, Julian Beck explique même la difficulté de se mettre en retrait, de quitter sa position d'initiateur pour laisser à chacun la place de s'exprimer ${ }^{11}$. Cela est toutefois nécessaire : selon eux, un.e artiste ne peut pas prêcher une parole qu'il ne tente pas lui-même de mettre en action. Ce n'est pas l'ambition de Gwenaël Morin ni de sa troupe, qui va recruter les vingt-quatre comédien.ne.s manquant.e.s.

12 L'objectif du spectacle, selon le Living Theatre, était de préparer les artistes à travers le processus de création-tout comme les spectateur.ice.s grâce à la représentation-à la 
révolution. Julian Beck expliquera les positions de la troupe en ces termes « D'un côté, nous rêvons d'un théâtre si puissant que les gens le quittent pour s'engager dans la révolution, d'un autre côté, on espère que le travail que nous accomplissons au théâtre sert les besoins du public et le guide vers une action politique et économique qui les aide à sortir de leur camisole de force ${ }^{12} »$. Gwenaël Morin ne tente pas, en décidant de mettre en scène ce spectacle, de créer une communauté anarchiste éphémère composée d'artistes amateur.ice.s. Il conserve son statut de metteur en scène et fait appel à des comédien.ne.s alors rémunéré.e. $\mathrm{s}^{13}$. D'un point de vue dramaturgique, cela a un impact sur le spectacle, qui passe de cinq à six heures de représentation lorsqu'il est joué par le Living Theatre à deux heures de représentation avec la troupe de Gwenaël Morin. Le metteur en scène s'en explique, "pour pouvoir mener ces scènes d'impro[visations], il faut avoir beaucoup travaillé ensemble avec les acteurs, sur d'autres choses, et ça, nous, on n'avait pas les moyens ${ }^{14}$ ». Les scènes d' « actions » sont donc raccourcies. Les différences de jeu entre l'originale et la version de Gwenaël Morin proviennent de cette non-ambition politique, qui a coupé court à l'expérimentation longue durée et donc aux Actions.

Paradise Now était construit autour d'improvisations spontanées, de la part des artistes et $\mathrm{du}$ public ${ }^{15}$. Le Living n'était dans une recherche esthétique qu'à partir de leur propos. Judith Malina dira d'ailleurs « concevoir le théâtre comme un acte politique et le contenu comme instigateur de la forme ${ }^{16} »$. Comment alors représenter un spectacle qui se veut révolutionnaire si sa substance provoque les moyens de sa mise en scène, mais que les artistes ne sont pas dans une optique politique? C'est là tout le dilemme du projet de Gwenaël Morin, qui nous mène à nous interroger sur une problématique nécessairement politique: la relation du spectacle à l'institution. Pour le Living Theatre, la nécessité de créer une société anarchiste participait aussi de leur volonté de ne plus faire partie du monde capitaliste et de ne plus être dans des rapports financiers. Chez Gwenaël Morin, les comédien.ne.s sont payé.e.s au cachet. Le spectacle reçoit deux sources de financement pour sa création, l'une du théâtre du Point du Jour et l'autre du CDN Nanterre-Amandiers. Il s'inscrit alors de fait dans la catégorie du théâtre subventionné français, et s'oppose directement à la pratique théâtrale du Living Theatre qui tentait à tout prix de s'extraire de ce théâtre dominant et de ce qu'il représente $^{17}$. Le Living, qui dénonçait le théâtre bourgeois et la marchandisation de la culture voit aujourd'hui son spectacle rejoué au cœur de l'institution théâtrale française, le centre dramatique national Nanterre-Amandiers, par un metteur en scène lui-même bien installé, alors directeur d'un théâtre lyonnais. Plus encore, tandis que la révolution de juillet 1968 portait en elle la revendication d'un théâtre gratuit et ouvert à tou.te.s, le politique est là encore désamorcé : les représentations à NanterreAmandiers sont payantes ${ }^{18}$.

Mais alors, comment recevoir cette proposition théâtrale? Si Gwenaël Morin se met en difficulté en décidant de mettre en scène cet héritage colossal et révolutionnaire, il ne faut pas oublier ses objectifs artistiques propres, et regarder à tout prix cette mise en scène à travers le regard du Living. Toute l'ambition de Gwenaël Morin est de nous faire nous demander «pourquoi?» Pourquoi, aujourd'hui, cinquante ans après les révolutions de mai et juin 1968, mettre à nouveau en scène ce spectacle? Qu'est-ce que cela fait aux spectateurs et spectatrices? Quelle place donne-t-on aujourd'hui aux idéologies politiques révolutionnaires? À l'anarchisme? À la liberté individuelle et collective? L'artiste n'a que peu de connaissances sur l'anarchisme, sur le théâtre radical américain des années 1970 et sur le Living Theatre. Son travail est de se mettre 
" en empathie totale avec ce qui est écrit », dit-il. «Du coup, je n'ai aucun exercice critique. Je n'utilise pas les pièces, quelles qu'elles soient comme une espèce de support de réflexion ou comme une manière... Je n'essaie pas de les orienter dans un sens qui serait conforme. [...] Je prête le flanc à tous les stéréotypes et à toutes les idées reçues qui sont consécutives de la part superficielle de ma propre culture en fait, et c'est d'une certaine ce qui m'intéresse au bout du compte ${ }^{19} »$. Gwenaël Morin soulève ici une interrogation bien plus importante que celle de savoir comment mettre en scène aujourd'hui les pièces d'hier en conservant leur substance et leur caractère subversif lorsque le contexte politique et social a évolué. Il ne s'agit plus de se demander comment traduire les événements politiques du passé, ou comment réadapter une pièce de théâtre politique historique, mais plutôt et simplement de se demander l'objectif que l'on fixe au théâtre lui-même.

Le pari semble en fait réussi: si la transformation du public est difficilement perceptible, ce spectacle soulève des interrogations essentielles, tant au monde du théâtre qu'à la vie politique du spectateur ou de la spectatrice. Il interroge la nécessité de réactiver les œuvres du passé, voire de les réadapter. Gwenaël Morin ici n'effectue ni une reconstitution historique - il n'a pas d'ambition scientifique dans la mise en scène de ce spectacle - ni une réadaptation. Cette dernière aurait nécessité une nouvelle traduction théâtrale : le contexte de représentation n'est plus le même, les interprètes sont différent.e.s, le public a une autre perception du théâtre et du subversif. Réadapter cette pièce aurait signifié en conserver sa substance politique et son ambition révolutionnaire pour que le public les perçoive. En effet, plutôt qu'une reconstitution ou une réadaptation, le metteur en scène a produit un hommage, qui, bien que dépolitisé, aura au moins eu le mérite de remettre sur le devant de la scène cette troupe américaine historique et ses luttes. À défaut d'une transformation du spectateur ou la spectatrice, cette version aura contribué à modifier les relations de travail au sein de l'équipe artistique. Selon Gwenaël Morin lui-même, ce spectacle l'a transformé et sa mise en scène $\mathrm{a}$ eu un impact fort sur les comédien.ne.s « C'est la première fois que je monte une pièce qui me transforme autant dans sa pratique. Quand on [a] mont[é] la pièce, ce que ça a généré entre nous, dans l'équipe [...], une espèce de... les gens [de l'équipe] étaient tous amoureux les uns des autres ${ }^{20} »$. Il ne s'agit pas pour l'artiste ici de promouvoir une nouvelle société, mais bien de voir comment celle dans laquelle on vit réagit cinquante ans plus tard à ces discours. L'aspect révolutionnaire de ce spectacle historique se cachait peut-être, depuis le début, non pas dans sa représentation, mais dans son processus de création.

\section{NOTES}

1. A ce sujet, consulter: É. Jouve, Avignon 1968 et le Living theatre: mémoires d'une révolution, Montpellier, Éditions Deuxième époque, 2018.

2. Dramaturge, scénographe et metteur en scène français, il dirige depuis 2014 le centre dramatique national Nanterre-Amandiers. 
3. Nous faisons ici référence au Théâtre permanent de G. Morin et ses mises en scène de Racine, Molière, Sophocle, Tchekov, Shakespeare, etc.).

4. J. Beck, La vie du théâtre, A. Vander et F. Vander (trad.), Paris, Gallimard, 1978.

5. J.-J. Lebel, Entretiens avec le living theatre, Paris, Editions Pierre Belfond, 1969.

6. S. Vendeville, Le Living Theatre : de la toile à la scène : 1945-1985, Paris, l'Harmattan, 2007.

7. J. Malina et J. Beck (éd.), Paradise now: collective creation of the Living Theatre, New York, EtatsUnis d'Amérique, Vintage Books, 1971.

8. Voir la traduction distribuée durant le spectacle de Paradise Now par Elsa Rooke pour Gwenaël Morin, p. 2.

9. J.-J. Lebel, Entretiens avec le living theatre, op. cit., p. 164.

10. J. Malina et J. Beck (éd.), Paradise now, op. cit. «La pièce Paradise Now n'est pas une propriété privée : / il n'y a pas de droits de représentation à payer :/c'est gratuit :/pour toute communauté qui souhaiterait la jouer. " traduction personnelle de l'extrait suivant: "The play "Paradise Now" is not private property:/there are no performance royalties to pay:/it is free:/for any community that wants to play it ».

11. Les créations collectives commencent avec Mysteries and smaller pieces, en 1964, à partir de leur exil en Europe. Concernant la difficulté de la déhiérarchisation et de la création collective, voir P. Biner, Le Living Theatre : histoire sans légende, Lausanne, Suisse, La Cité éd., 1968, p. 172.

12. J.-J. Lebel, Entretiens avec le living theatre, op. cit., p. 13.

13. «Audition pour Re-Paradise, spectacle de Gwenael Morin @ Théâtre des Amandiers/ Nanterre, Paris [du 11 au 13 avril]», sur paris.carpe-diem.events, https://paris.carpediem.events/calendar/6398678-audition-pour-re-paradise-spectacle-de-gwenael-morin-at-thtre-des-amandiers-nanterre/, s. d.

14. Interview personnelle avec Gwenaël Morin, le 02/11/2019 à Lyon, p. 19.

15. Pour les réactions de spectateurs, voir J.-J. Lebel, Entretiens avec le living theatre, op. cit.

16. Id., p.9.

17. "Judith et moi [écrivit Julian Beck en 1962], avons travaillé pour construire une compagnie sans les maniérismes, les voix, la bonne élocution, le camouflage des acteurs qui imitent le monde de la Maison Blanche, qui rejouent les petits malheurs et les souffrances de la bourgeoisie » in J. Beck, La vie du théâtre, op. cit., p. 20-21.

18. Notons que la représentation au Théâtre du Point du Jour était gratuite, comme beaucoup de représentations de G. Morin lors de ses cinq années de direction de structure.

19. Interview personnelle avec Gwenaël Morin, le 02/11/2018 à Lyon, p. 6.

20. Interview personnelle avec Gwenaël Morin, le 02/11/2018 à Lyon, p. 16.

\section{RÉSUMÉS}

Critique du spectacle Re-Paradise de Gwenaël Morin mis en scène dans le cadre du festival «Mondes Possibles » du centre dramatique national Nanterre-Amandiers pour les 50 ans de mai 1968.

Review of Gwenaël Morin's Re-Paradise presented at the national dramatic center of Theatre Nanterre-Amandier for the Festival "Mondes Possibles" celebrating the fiftieth anniversary of May 1968. 
INDEX

Mots-clés : Théâtre politique, anarchisme, subversif, réactivation, reconstitution, réadaptation, Gwenaël Morin, Living Theatre, CND Nanterre-Amandiers, Théâtre du Point du jour, théâtre révolutionnaire américain, Paradise Now, 1968

Keywords : Political theatre, anarchism, subversive, reactivation, reenactement, adaptation, Gwenaël Morin, Living Theatre, CND Nanterre-Amandiers, Théâtre du Point du jour, American revolutionary theatre, Paradise Now, 1968

Thèmes : Theater

\section{AUTEUR}

\section{CAMILLE MAYER}

Doctorante contractuelle en études théâtrales, EDESTA, EA1573 Scènes du monde Université Paris 8 Vincennes/Saint-Denis

Camille.mayer@hotmail.fr 